\title{
XLI.
}

\section{Chemische Untersuchung der Mineralquelle von Nieder-Langenau in der Grafschaft Glatz.}

\author{
Von \\ Dr. Theodor Poleck.
}

In dem an Naturschönheiten reichsten und klimatisch begünstigsten Theile der Grafschaft Glatz ist westlich von dem Gebirgsstock des Schneeberges auf dem linken Ufer der Neisse, 1330 Fuss über der Ostsee in reizender Umgebung die Mineralquelle von Nieder-Langenan gelegen. Das Thal der Neisse wird hier von Pläner - und Quadcrsandstein gebildet, der sich auf beiden Seiten des Flusses zu nicht unbedeutenden Höhen erhebt. Der Glimmerschiefer und Gneuss, welche das Gebiet der Kreideformation begrenzen und von dieser unnittelbar überlagert werden, durchbrechen letzlcre an einzelnen Orten und treten namentlich bei Nieder-Langenau an beiden Seiten der Neisse iuselartig hervor, Vereinzelte Basaltkegel finden sich in einer Entfernung von zwei Meilen bei Landeck.

Dic Quelle selbst entspringt in einem alten Stollon aus Glimmerschiefer, über welchem eine Lehmschicht lagert. Sie kann nicht unmittelbar an ihrem Ursprunge benutzt werden, sondern sie wird von $\mathrm{da}$ in Rohren nach der nahen Trinkhalle und dem Badehause geleitet. Hier kommt sie als eine gesittigte Auflösung der Kohlensăure in Wasser an, über welcher einc bedeutcnde, aber stets wechselnde Kohlensãure-Schicht sich befindet, welche in neuester Zeit ihre Benutzung als Gasbad gefunden hat.

Die Quelle liefert nach früheren Ermittelungen durchschniltlich in 24 Stunden 57,600 Quart Wasser, ungerechnet der Wassermenge, welche nach einer andern Seite hin unbenutzt abniesst. Ihre Temperatur ist constant zwischen $9^{\circ}$ und $10^{\circ} \mathrm{C}$. an 25. Mai und am 2. September 1850 wurde sie bei einer Lufltemperatur von $20^{\circ} \mathrm{C}$. und von $15,4^{\circ} \mathrm{C}$. zu $10^{\circ} \mathrm{C}$. beols achtet, während frühere Beobachtungen bei $\mathbf{1}^{0} \mathrm{C}$. Luftemperatur sic $9^{\circ} \mathrm{C}$. fanden. Schwankungen über diese Grenzen hinats sind nie beobachtet worden.

Journ. f. prakt. Chemie. LII. 6 
Dos frisch geschöpte Wasser ist krystallıell, perlt stark in kleinen Bläschen und hat einen. angenehmen, erfrischenden, sãuerlich-prickelnden, kaum salzigen Geschmack, der lurch seine Eigenthümlichkeit sogleich den bedeutenden Eisengehalt verrāth. Es ist geruchlos und rơthet vorübergebend Lackmuspapier. In offnen Gefüssen der Luft ausgesetzt entwickelt es nur langsam seine Kohlensãure, verlicrt jedoch in kurzer Zeit den grössten Theil des aufgelösten Eisens als Eisenoxyd. Die gleiche Zersetzung findet nach einiger Zeit in den Flaschen statt, in denen es versandt wird. Beim Kochen trübt es sich urter reichlicher Entwickelung von Kohlensāure, wobei das Eisen und die Erdsalze sich vollstāndig abscheiden; das Wasser schmecht und reagirt dann deutlich alkalisch.

Die Analyse zerfiel in die Untersuchung des bei längerem Erhitzen des Wasser's sich bildenden Niederschlogs und der von demselben abfiltrirten Flüssigkeit. In ersterem wurden nach bekanuten Methoden Kalk, Magnesia, Eisen- und Manganoxydul als kohlensaure Salze, phosphorsaure Thonerde, Kieselerde und Spuren von Arsenik nachgerwiesen. Die Versuche, Fluor und Strontian sowie Quell - und Quellsatzsāure zu entdecken, zeigten die Abwesenheit dieser Körper. In der abfiltrirten Flüssigkeit wurden Kali, Natron, Lithion, Chlor, Schwefelsăure, Kohlensãure und cxtractire organische Substanz gefunden. Jod, Brom, Salpetersãure und Ammoniak konnten nicht entdeckt werden. Diese Kürper, sowie Arsenik, wurden in dem Rückstande gesucht, welcher durch vorsichtiges Verdampfen von 116 Pfl. Mineralwassel auf wenige Plunde erlalten worden war. Letzterer wurde auch für die quantitative Bestimmung derjenigen Bestandtheile les Wassers benutzt, welche nur in geringer Menge in ihm rorkommen. Arsenik konnte im Rückstande des abgedamptten Wassers nicht nachgewiesen werden, dagegen war er in ãusserst geringer, quantitativ nicht bestimmbarer Menge in den Absătzen vorhanden, welche sich an der Quelle und in der Röhrenleitung billen. Nach schweren Metallen wurde vergebens gesucht.

\section{Bestimmung des specifischen Gexichts.}

Das Verhältniss der Gewichte gleicher Volumina destillirten Wassers und des Mineralbrunnens war bei $6,5^{\circ} \mathrm{C}$. wie 20,488 : 
Mineralquelle von Nieder-Langenan in der etc. 355 20,516, woraus sich das specifische Gervicht des letzteren zh 1,00136 ergiebt.

1. Beslimmung des Gesammiquantums der fixen Bistandtheile.

a) $233,856 \mathrm{Gr}$, des Mineralwassers hinterliessen nach dem Verdampfen zur Trockne und gelindem Erhitzen 0,1963 Gr. Rückstand $=0,08394$ p. C.

b) $893,068 \mathrm{Gr}$. gaben $0,7415 \mathrm{Gr}$. $=0,083028 \mathrm{p}$. C. Rückstand; von diesem Josten sich beim Uebergiessen mit Wasser $0,1825 \mathrm{Gr}$. $=0,020435 \mathrm{p}$. C. auf, es blieben mithin ungelist $0,5590 \mathrm{Gr}$. $=0,062593$ p. C.

\section{Bestimmung des Chlors.}

a) $876,96 \mathrm{Gr}$. des Nineralwassers gaben $0,0195 \mathrm{Gr}$. Chlorsilber $=0,000547$ p. C. Clslor.

b) $876,96 \mathrm{Gr}$. gaben ferner $0,0192 \mathrm{Gr}$. Chlorsilber $=$ 0,000540 p. C. Chlor.

Mittel der Versuche $=0,000543$ p. C. Chlor.

\section{Bestimmung der Schwefelsäure.}

a) $439,964 \mathrm{Gr}$. Wasser gaben $0,0171 \mathrm{Gr}$. schwefclsauren Baryt $=0,001335$ p. C. Sclıwefelsäure.

b) $350,852 \mathrm{Gr}$. gaben 0,0138 Gr. schwefelsauren Baryt $=$ $0,001351 \mathrm{p}$. C. Schrwefelsăure.

Mittel der Versuche $=0,001343$ p. C. Schwefelsäturc.

\section{Bestimmung des Chlornatriums.}

In 2 wurde gefunden, dass 100 Theile Nineralwasser 0,000543 p. C. Chlor enthalten. Diese sind lediglich an Natrium gebunden und bedürfen davon $0,00035 \mathrm{j}$ p. C. 100 Theile des Wassers enthalten mithin 0,000898 p. C. Chlornatrium.

\section{Bestimmung der Gesammimenge der Alkalien.}

Diese geschah nach zwei sich gegenseitig controlirenden Methoden.

a) Aus 1169,28 Gr. Wasser wurden auf bekannte Weise 0,2567 Gr. Chloralkalien, 0,1005 Gr. Kaliumplatinchlorid entsprechend 0,0306 Chlorkalium und 0,2787 Gr. schivefelsaures Natron erhalten, welche 0,2294 Gr. Chlornatrium entsprechen. 
Wenn man die gefundenen $0,0306 \mathrm{Gr}$. Chlorkalium von der Gesammtmenge der Chloralkalien abzieht, so Jleiben $0,2261 \mathrm{Gr}$. Chlornatrium übrig. Im Nittel sind also erhalten worden 0,2277 Gr. Chlornatrium, welche gleich sind 0,010321 p. C. Natrinmoxyl.

0,0306 Chlorkalium entsprechen 0,001635 p. C. Kali.

b) $893,068 \mathrm{Gr}$. Wasser wurden zur Trockne verdampft; der Büclistand mit Wasser ausgezogen, das Filtrat durch Salzsiuture in Chlormetalle und endlich durch Schwefelsiure in neutrale schwefelsaure Salze rerwandelt. Es wurden $0,2390 \mathrm{Gr}$. schwefelsaure Salze erhalıcu, welche nach ihrer Lösung in Wasser und Fallung mit Chlorluaryum 0,3825 Gr. schwefelsauren Baryt gaben, welcher 0,1314 Gr. Schwefelsäure enthält. Daraus berechncte ich nach den Formeln $N=\frac{S-(A \times 0,45916)}{0,10207}$ und $\mathrm{K}=\mathrm{A}-\mathrm{N}$, worin $\mathrm{d}$ ofleich dem Gemenge der schwefelsauren Allialien, $\mathbf{N}=\mathrm{dem}$ darin enthalteneu schwefelsauren Natron, $\mathbf{K}=$ dem schwefelsaturen Kali und $\mathbf{S}=$ dor darin enthaltenen Schwefelsăure ist, $-0,0268 \mathrm{Gr}$. schwefelsaures Kali $=0,001622$ p. C. Kali und 0,2122 Gr. schwefelsaures Natron $=0,010369$ p. C. Natron.

In a wurden exhalten 0,001653 p. C. Kali,
In b dagegen 0,001622 p. C. Kali. Ilittel der Versuche $=0,001637$ p. C. Kali.

It a wurden erhalten 0,010321 p. C. Natron. In b) dagegen $\quad \mathbf{0 , 0 1 0 3 6 9}$ p. C. Natron. Hittel der Versuche 0,010345 p. C. Natron.

\section{Bestimmung des schwefelsauren Kali.}

Die im Wasser vorbandene Schwcfelsäure ist lediglich an Fili gebunden. In 3 wurden im Mittel 0,001343 p. C. Schwefelsätre gefunden, welche 0,001581 p. C. Kali erfordern. In 5 wurden 0,001637 p. C. Kali gefunden, welche, wenn der unbe dentende Ueberschuss von 0,000056 p. C. vernachlässigt wird, nur als schwefelsaures líali im Wasser vorhanden sind.

Dic Menge des schwefelsauren Kali hetriagt daher 0,00292' p. C. In 56 , wurden $0,0268 \mathrm{Gr}$. $=0,003000$ p. C. scliwefelsaures Kali gefunden. 


\section{Bestimmung des kohlensauren Lithions.}

7539,4 Gr. Wasser wurden zur Trockne verdampft, der Rückstand schwach geglüht, wieder in Wasser gelōst und das Filtrat mit phosphersaurem Natron im Ueberschuss zur Trockne verdampft. Das erhaltene phosphorsaure Natron-Lithion wurde mit kaltem Wasser ausgewaschen und durch sein Verhalten vor dem Löthrohr als rein erkannt. Es wog $0,0090 \mathrm{Gr}$., welche 0,000037 p. C. kohlensaurem Lithion entsprechen. In einem ziveiten Versuche wurden aus $7539,4 \mathrm{Gr}$. des Wassers 0,0010 Gr. phosphorsaures Natron-Lithion erhalten, welche 0,000041 p. C. kollensaurem Lithion entsprechen. Mittel der Versuche $=0,000039$ p. C. kohlensaures Lithion, welche 0,000024 p. C. liohlensäure enthalten.

\section{Bestimmung des kohlensauren Nalrons.}

$893,068 \mathrm{Gr}$. des Mineralwassers wurlen zur Trockne verdamph, der Rückstand mit Wasser aufgenommen und im Filtrat die kohlensauren Alkalien in Chlormetalle verivandelt. Durch Abdampfen der Lösung and Glühen des Rückstandes wurden 0,1975 Gr. eines Salzrūckstandes erhalten, wclcher grleich ist

$$
=0,022114 \mathrm{p} \text {. c. }
$$

darunter befinden sich nach 4 Kochsalz

0,000898 p. C.,

nach 6 schwefelsaures Kali 0,002924 p. C.,

von obiger Summe daher abzuziehen
es bleiben daher $\frac{0,003822 \text { p. C. }}{0,018292 \text { p. C. }}$

Chlornatrium übrig, welche 0,016584 p. C. kolılensaurem Nal'on entsprechen, von welcher Ilenge jedoch nach 7 noch 0,000039 p. C. kohlensaures Lithion abzuziehen sind.

Das Wasser enthält mithin $0,016545 \mathrm{p}$. C. kohlensaures Natron. In 5 a wurden nach $\Lambda$ bzug des Chlornatriums 0,016840 p. C. Kohlensaures Natron erhalten, wovon ebeufalls noch 0,000039 p. C. kohlensaures Lithion abzaziehen sind, so lass 0,016801 p. C. kohlensaures Natron übrig bleiben.

Mittel der Versuche $=0,016672$ p. C. kohlensaures Natron, welche 0,006924 p. C. Kohlensätre entlialten. 
9. Controle der in Wasser löslichen Bestandtheile.

Nach 1 b beträgt die Menge d. lósl. Bestandtheile 0,020435 p.C. nach 4 die Menge des Chlornatriums 0,000898 p. C. nach 6 die Menge des schwefels. Kali 0,002924 " nach 7 d. Menge d. kohlens. Lithions 0,000039 , mach 8 d. Menge d. kohlens. Natrons 0,016672 , Summe 0,020533 p.C.

10. Bestimmung der Kieselerle.

a) $910,455 \mathrm{Gr}$. des Wassers gahen $0,0485 \mathrm{Gr}$. Kieselerde $=0,005327$ p. C.

b) $1050,442 \mathrm{Gr}$. Wasser gaben $0.0575 \mathrm{Gr}$. $=0,005473$ 1). C. Kieselerde.

Mittel der Versuche $=0,005400$ p. C. Kieselerde.

11. Bestimmung tes kohlensauren Eisenoxyduls.

Bei dieser Bestimmung war es nothwendig, den gesammten Inlalt der einzelnen Flaschen zur Analyse zu verwenden, da auch bei völlig klar geschöpftem Wasser und gutem Verschlıss der Flaschen sich schon nach wenig Tagen ein Theil des Eisens als Eisenoxyd niedergeschlagen hatte. Das fest am Boden der Flasche haftende Eisenoxyd wurde mit wenig Salzsãure heraus gevaschen.

a) 910,455 Gr. IVasser gaben $0,0215 \mathrm{Gr}$. $=0,002361 \mathrm{p}$. C. Eisenoxyd.

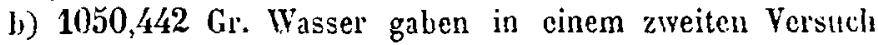
$0,0275 \mathrm{Gr}$. $=0,002617$ p. C. Eisenoxyd.

c) Das Eisenoxyd wurde noch in dem Nicilersclılage bestimmt, der bei der Bestimmung der Gesammtınenge der Kohlensãure an der Quelle crhalten worten war.

3,5935 Gr. dieses Niederschlags, entsprecheud 515,3 Gr. Mineralwasser, gaben $0,0145 \mathrm{Gr}^{2}=0,002813 \mathrm{p}$. C. Eisenoxyd.

Mittel der Versuche $=0,002597$ Eisenoxyd, welche entsprechen 0,003765 p. C. kohlensaurem Eisenoxyclul und diese enthalten 0,001428 p. C. Kohlensāure.

12. Bestimmung der phosphorsauren Thonerde.

Die Thonerde wurde vom Eisenoxyd durch $\mathrm{K}_{\text {aliflüssigkeit }}$ getrennt und aus dieser durch Salmiak gefallt. Sie enthielt Phosphorsäure, welche durch molybdänsaures Ammoniak nach- 
gewiesen wurde, ihrer geringen Menge wegen jedoch nicht quantitativ bestimmt werden konnte.

a) $910,45 \mathbf{j}$ Gr. des Wassers gaben $0,0010 \mathrm{Gr}$. Thouerde $=0,000109$ p. C.

b) 1050,442 Gr. des Wassers gaben 0,0015 Gr. Thonerde $=0,000142 \mathrm{p}$. C.

Mlittel der Versuche $=0,000125$ p. C. phosphors. Thonerde.

13. Bestimmung des kohlensauren Kalks.

a) $910,455 \mathrm{Gr}$. Wasser gaben $0,3310 \mathrm{Gr}$. $=0,036355 \mathrm{p}$. C. kohlens. Kalk.

b) 1050,442 Gr. gaben $0,3840 \mathrm{Gr}$. $=0,036556 \mathrm{p}$. C. kohlensauren Kalk.

Mittel der Versuche 0,036455 p. C. kohlensaurcr Kalk, welche 0,016042 p. C. Kohlensāure enthalten.

14. Bestimmung der kohlensauren Magnesia.

910,455 Gr. Wasser gaben 0,214 Gr. phosphorsaure Magnesia, welche 0,017798 p. C. kollensaurer Magnesia entsprechen und diese enthalten 0,009187 p. C. Koblensãure.

Ein zweiter Versuch gab ein ungenaues Resultat.

15. Bestimmung des kohlensauren Mranganoxyduls.

$\mathrm{Zu}$ dieser Bestimmung wurden 9,2483 Gr. des in Wasser unlőslichen Rückstandes, welcher durch Eindampfon von 116 Pfd. Mineralwasser erhalten worden war, verwandt.

Diese Menge entspricht 15920 Gr. Wasser. Eisen und Mangan wurden durch bernsteinsaures Ammoniak getrennt und das Manganexydul durch kohlensaures Natron gefält.

$15920 \mathrm{Gr}$. des Nineralwassers gaben $0,0535 \mathrm{Gr}$. Manganoxyduloxyd, welche 0,000505 p. C. kolhlensaurem Hanganoxydul entsprechen. Diese enthalten 0,000193 p. C. Kohlensäure.

16. Controle der in Wasser unlöslichen Beslandheile.

Nach $1 \mathrm{~b}$ wurde die Gesammtmenge der in Wasser unlöslichen Bestandtheile gefunden Nach 10 betrăgt die Kieselerde $\quad 0,005400$ p. C.

"11 die Mlenge des Eisenoxyds 0,002597 "

" 12 d. M.d. phosphors. Thonerde 0,000125 "

" 13 u. M. d. kohlens. Kalks 0,036455 ",

" 14 d.M.d. kohlens. Magnesia 0,017798 "

" 15 d.M.d.kohlens.Manganoxydul 0,000505

Summa 0,062880 p.C. 
17. Schlusscontrole.

Die durch die Analyse gefundenen löslichen Bestandtheile des Wassers sind

Die unlōslichen

Wir fanden in 1 a direct

$$
\begin{aligned}
=0,020533 \text { p.C. } & 0,062880 \text { p.C. } \\
\text { Summa } & \mathbf{0 , 0 8 3 4 1 3} \\
& 0,083940 \text { p. C. }
\end{aligned}
$$

\section{Bestimmung der Kohlensäure im Ganæcn.}

Zur Bestimmung der Kohlensăure wurde ein Stechheber von bekanntem Inhalt an der Quelle unter Wasser gefüllt und scin Inhalt in eine Flasche entleert, welche eine Auflösung von Chlorcalcium und Ammoniak enthielt. Die wohlverschlossenen Flaschen wurden in kochendem Wasser erhitzt und der gefällte kohlensaure Kalk rasch abfittrirt und gewogen. In den erhaltenen Niederschlügen wurde dann nach bekannten Methoden die Kohlensãure bestimmt.

Die Füllung der Flaschen geschah am 25. Mai 1850 zu dev Zeit, als das Wasser für die Analyse geschöpft wurde.

1. 200,452 Gr. Wasser gaben $1,3883 \mathrm{Gr}$. Niederschlag,

2. 200,452 Gr. " " 1,3970 Gr. "

3. $200,452 \mathrm{Gr}$. " " 1,4010 Gr. "

4. $200,452 \mathrm{Gr}$. " " $1,3880 \mathrm{Gr}$. "

Mittel $1,3936 \mathrm{Gr}$. $=0,69522 \mathrm{p}$. C.

1. $0,6565 \mathrm{Gr}$. dieses Niederschlags entliciten $0,2585 \mathrm{Gr}$. Kohlensăure $=39,37$ p. C. lensäure.

2. $0,7100 \mathrm{Gr}$. gaben ferner $0,2835 \mathrm{Gl}$. $=39,92$ p. C. KuhMittel der Versuche 39,64 p. C. Kohlensäurc.

0,69522 Gr. des Niederschlags, entsprechend 100 Theilen Wassel, liefern demnach 0,275585 p. C. Kohlensäure.

Schon im Jahre 1849 im Juli und September war die Gesammtmenge der Kohlensäure auf gleiche Weise von mir ermittelt worden. Das erste Mal wurden 0,275338 p. C., das zweite Mal 0,272182 p. C. Kohlensăure gefunden. 
19. Bestimmung der freien Kohlensäure.

Die Gesammtmenge der Kohlensãure ist nach 18

Gebund. Kohlensãurc an Lillion n. 7 0,000024 p. C.

$$
=0,275585 \mathrm{p} . \mathrm{C} \text {. }
$$

$$
\begin{aligned}
& \text { an Natron n.8 } 0,006924 \text {," } \\
& \text { an Kalk n. } 130,016042 \text {, } \\
& \text { an Magnesia n. } 14 \text { 0,009187," } \\
& \text { an Eisenoxydul n.11 0,001428," } \\
& \text { an Manganoxydul n. } 150,000193,-0,033798 \text { p. C. } \\
& \text { bleibt für freic Kohlcnsanture } \mathbf{0 , 2 4 1 7 8 7} \text { p.C. }
\end{aligned}
$$

Da jedoch sämmtliche kohlensaure Salze des Wassers als Bicarbonate vorhanden sind, so ist die doppelte Gewichtsmenge der gebundenen Kolılensüure ron ihrer Gesammtmenge abzuzichen; es bleiben daher 0,207989 p. C. freic Kohlensäure übrig.

0,207989 Gr. Kohlensāure entsprechen bei $0^{0} 105,205 \mathrm{CC}$ und sind bei der Temperalur der Quelle $10^{\circ} \mathrm{C} .=109,06 \mathrm{CC}$.

20. Zusammenstellung der Resulfate.

I. 10,000 Gewichtstheile Jineralwasser enthalten:

A. Fixe Bestandtheile

1. in wăgbarer Ilenge vorhanden:

Chlornatrium

Schwefclsaures Kali

Kohlensaures Natrou

Kohlensaures Lithion

Kohlensaurer Kalk

Kohlensanre Magnesia

Kohlensaures Eisenoxydul " Manganoxydul

Phosphorsaure Thonerde

Kieselerde

2. in unwägharer Menge vorhanden:

Arsensiures Eiscnoxyd

Extractive organische Materie

Summe der fixen Bestandthcile 8,4581 Theile

B. Flüchtige Bestandtheile:

An Bikarbonatc gebund. Kolllens. 3,3798 Theile

Freie Kohlensänre

0,0898 Theile

0,292 1

1,0672,

0,003 ! ,

3,6455

1,7798

0,3765

0,0505

0,0125

$0,5 \mathbf{1 0 0}$

Summe aller Bestandtheile 32,6368 Theile 
Nach dem gefundenen specifischen Gewichte entsprechen 100,136 Gewichtstheile Mineralwasser 100 Voluntheilen. 100 Gewichtstheile entsprechen aber bei der Temperatu der Quelte 109,06 CC. Kohlensäure, 100,136 Theile also 109,208 CC. Kohlensäure. 100 Volumtheile Mineralwasser enthalten demnach 109,208 Volumtheile Kohlensãure. Da kaltes Wasser ein Volumen Kolılensäure auflöst, so ist der Langenauer Mineralbrunnen eine gesălligte Auflōsung von Kohlensāure in Wasser.

Herr Professor Dr. Duflos, welcher in Auftage der höniglichen Regierung zu Breslau im September des Jahres 1850 ebenfalls die Analyse dieser Mineralquelle ausführte, theilte mir die Resultate derselben mit, um sie mit den meinigen zu verülfentlichen.

Ein Pfund Wasser $=16$ Unzen $=7680$ Gran enthālt

1. Fixe Bestandtheile nach meiner Analyse nachProf.Dr.Duflo's

Juni $1850 . \quad$ September 1850.

Chlornatrium $\quad 0,06896$ Gran. $\quad 0,06912$ Gran.

Schwefelsaures Kali $\quad 0,22456^{\circ}$ "

Kohlensaures Natrou 1,28041 " 1,27480 ,

$\begin{array}{llll}\text { Kohlensaures Lithion } & 0,00299 & " & - \\ \text { Kohlensauren Kalk } & 2,79974 & \text { ", } & 2,79552\end{array}$

Kohlensaure Magnesia 1,36688 ", J,38240 ",

Kohlensaures Eisenoxydul 0,28915 " $\quad 0,28876$ ",

Kohlens. Manganoxylul 0,03878 " 0,03072 "

Phosphorsaure Thonerde $0,00960 "$ Spuren

Kiesclerde

Arsenige Sinre

$0,41472 " 0,114 \tau_{2}$,

2. Flüchtige Bestandtheile

$\frac{\text { Spuren }}{6,49579} \quad$ etwa 1 Gran in 11328 Pfd

An Bikarbonate gebund. Kohlens. 2,59568 Gran. I

Freje Kohlensäure 15,97355

21,40416 Gran.

Summe aller Bestandtheile 25,06502 Gran. 27,88106 Gran.

15̆,97355 Gran Kohlensäure entsprechen bei der Temperatur der Quelle 28,508 Kubikzollen.

Im Juli 1849 wurden 28,47 Kubikzoll und in September 1849 27,89 Kubikzoll Kohlensăure gefunden. Die Schwankungen im Gelıalt an freier Koblensãure betragen also in Laule eines Jahres noch nicht cinen halben Kubikzoll.

Wenn die so gewonnenen Resultate mit den früheren Analysen von Dr. Günther, Medicinal-Assessor F ischer und 
Professor Dr. Fischer*) verglichen werden, so stellt sich mit Vebergehung einer Analyse von Trommsdorf $\mathrm{f}^{* *}$ ), der ein entschiedener Fchler zu Grunde liegt, heraus, dass diese Quelle in den dreissig Jalıren seit ihrer ersten Untersuchung sich wenig oder gar nicht in ihren Bestandtheilen geändert hat.

Die Hinernlquelle von Nieđler-Langenan gehört mithin zu den alkalisch-crdigen Eisen-Situcrlingen und zeiclmet sich nainentlich durch ihren bedeutenden Gelalt an freier Kohlensäure und die nicht muleträchtliche Menge von kuhlensaurem Eisenoxydul aus, während Chlormetalle und scliwefelsaure Salze fast găuzlich fehlen. In ihrer Zusammensetzung zeigt sie eine bemerkenswerthe Achnlichkeit mit den berühmten Stablquellen von Spaa ${ }^{* * *}$ ), namentlich dem Pouhon, mit denen sie auch in der Temperatur vollstindig überrinstimmt. Iangenau besitzt jedoch in seiner Quclle eincn ungleich grösseren Rcichthum an freier Kohlensāure, während der Gelhalt an kohlensaurem Eisen- und Manganoxydul von Span beinalee erreicht wird.

Für die medicinische Anwendung von Nieder-Langenau dürfte noch zu erwähnen sein, dass eine vortreflliche Moorwiese Veranlassung zu einem bequem eingerichteten Moor-Bade gegeben liat, welches schon in ausgedelmter Weise und mil günstigen Erfolgen benutzt worlen ist.

*) Pliysikalisch-medicinische Darstellung der bekannten Heilquellen von Dr. E. Osann, p. II. p. 383. - Das Bad Nieder-Langenau in der Grafschaft Glatz mit dem Jahreshericht fïr $184^{\mathrm{J}}$ von Dr. Wascmanu. Bresiau von Trewendt und Granier. p. 8. - Schlesische Provinzialblätter 1842. p. 355 .

**) Trom ms dorf Journal. 5. B. 1. Stïck. p. 319. 18:21.

***) Osann, physikalisch-medicinjsche Darstellung der bekaunten Heilquellen. p. II. p. 851 . 